This item was submitted to Loughborough's Research Repository by the author.

Items in Figshare are protected by copyright, with all rights reserved, unless otherwise indicated.

\title{
Optimal positioning of communication relay unmanned aerial vehicles in
} urban environments

PLEASE CITE THE PUBLISHED VERSION

http://dx.doi.org/10.1109/ICUAS.2016.7502562

PUBLISHER

(C) IEEE

VERSION

AM (Accepted Manuscript)

LICENCE

CC BY-NC-ND 4.0

REPOSITORY RECORD

Ladosz, Pawel, Hyondong Oh, and Wen-Hua Chen. 2016. "Optimal Positioning of Communication Relay Unmanned Aerial Vehicles in Urban Environments". figshare. https://hdl.handle.net/2134/22719. 


\title{
Optimal Positioning of Communication Relay Unmanned Aerial Vehicles in Urban Environments
}

\author{
Pawel Ladosz, Hyondong Oh, and Wen-Hua Chen
}

\begin{abstract}
This paper proposes a method of finding the optimal position of unmanned aerial vehicles (UAVs) functioning as a communication relay node to improve the network connectivity and communication performance of a team of ground nodes/vehicles. A three-dimensional complex urban environment containing many buildings is considered where the line-of-sight between ground nodes is often blocked. The particle swarm optimisation is used to find the optimal UAV position using three different communication performance metrics depending on the requirement. Numerical simulations are performed to show the advantage of using relay UAVs and the specific metric in sample scenarios. An indoor proof-ofconcept experiment is also performed to show the feasibility of the proposed approach in a real time.
\end{abstract}

\section{INTRODUCTION}

Ensuring communication between ground mobile agents/nodes reliably in urban environments is a quite challenging problem due to the limited bandwidth and effective range of the communication equipment and physical obscuration or occlusion [1]. In particular, the line-of-sight (LOS) between ground nodes is often obstructed by buildings and other terrain features; this affects the communication signal strength and consequently makes a ground-based radio communication unreliable and inefficient. Satellite communication (SATCOM) could be used instead, however, it has a limited spatio-temporal availability as the satellite follows a pre-planned orbit and the signal can still be blocked or delayed in urban environments. With the emergence of low-cost and robust small unmanned aerial vehicles (UAVs) as well as significant advancement of the communication technology, small UAVs can be used to address the urban communication problem. The UAVs can be optimally placed in the urban environment to improve communication performance between ground agents while functioning as airborne relay nodes. The relay UAVs can solve the majority of shortcomings of SATCOM and ground-based communication with its mobility in a sense that: i) UAVs can be deployed rapidly anytime and to anywhere; and ii) they can easily change position to mitigate the LOS block problem.

There are various approaches addressing the optimal positioning problem for UAVs to maintain network connectivity or improve communication performance of a networked team. They can be largely divided into [2], [3] in terms

This work was supported by the UK Engineering and Physical Science Research Council (EPSRC) under the Grant EP/J011525/1.

Pawel Ladosz, Hyondong $\mathrm{Oh}$ and Wen-Hua Chen are with the Department of Aeronautical and Automotive Engineering, Loughborough University, Loughborough, Leicestershire, LE11 3TU, United Kingdom \{p.ladosz, h.oh, w.chen\}@lboro.ac.uk of the performance metric used: i) model-based approaches where a known model of the communication environment is used to compute/predict the communication performance between the networked team members and ii) measurementbased approaches where online communication quality measurements such as received signal strength indication (RSSI) or the signal to noise ratio (SNR) are used.

For the model-based approaches, the number of connections to each UAV is used as the communication performance metric and it is combined with a flocking algorithm to maximise the number of connected nodes [4]. Ibrahim et al. [5] proposed the use of UAVs to improve second smallest eigenvalue of the Laplacian matrix of the network graph which leads to better network connectivity. Energy consumption and particle swarm optimisation (PSO) was considered in [6] where the UAV travels in a pattern which minimises the energy but maximises the UAV-ground nodes connection time. Several communication metrics were used to find the best position or trajectory of UAVs which maximises the communication performance of the team [7], [8].

For measurement-based approaches, the measured RSSI is used to create a force of attraction towards last known neighbour if the given number of connections to UAV drops to one or less [9]. Lee et al. [10] used the SNR while combining adaptive gradient estimation and efficient flying technique to provide a long endurance relaying capability. SNR was also used in [11] where gradient estimation was used to guide multiple UAVs to optimal position between two ground nodes. Similarly, Choi [12] proposed a manoeuvring scheme which minimises ergodic link capacity to each of the ground nodes.

Building upon the previous model-based approach [7], [8], this paper proposes the optimal positioning algorithm to deploy relay UAVs in a way to ensure network connectivity and maximise communication performance of the networked system, while connecting all team members with the minimum communication links and cost. Note that most aforementioned works only consider two dimensional (2-D) open field environment with no obstacles or buildings. In order to address this limitation, this work considers a 3-D urban scenario with several ground nodes scattered in the environment. The optimal position of relay UAV is found by the particle swarm optimisation (PSO) with three different communication performance metrics: global message connectivity (GMC), worst case connectivity (WCC) and modified global message connectivity (mGMC). The GMC and the WCC are used to improve the overall communication performance of the entire team and the poorest/weakest link 
only, respectively, as used in [7], [8]. Meanwhile, the mGMC proposed in this paper uses the certain number of poor connections to meet the desired communication requirements by combining the benefit of the GMC and the WCC.

The paper is structured as follows. Section 2 provides the problem scenario and the overview of the proposed approch. Section 3 provides the detailed description on the proposed optimal positioning algorithm. Section 4 shows the result of numerical simulations and an initial indoor flight experiment. Lastly, section 5 provides conclusions and future work.

\section{Scenario And Algorithm Overview}

A sample scenario is shown in Figure 1. The yellow dots represent ground nodes which are assumed to be performing their own mission, blue triangles represent communication relay UAVs, and cuboids are the buildings. The red lines represent best possible communication links connecting all ground nodes and UAVs. Several assumptions were made through this work. First, the knowledge of an urban environment and ground nodes positions is assumed to be known since the preliminary mission plan and the urban map can be obtained prior to the mission. Changes from the planned path are also assumed to be available in advance (for instance, the local change of the path can be reported to the ground control station before vehicle executes the new path) so that the updated ground vehicles positions can be used to calculate the new relay UAV position. Besides, it is assumed that if a line-of-sight between nodes is obstructed by buildings, communication quality is significantly reduced.

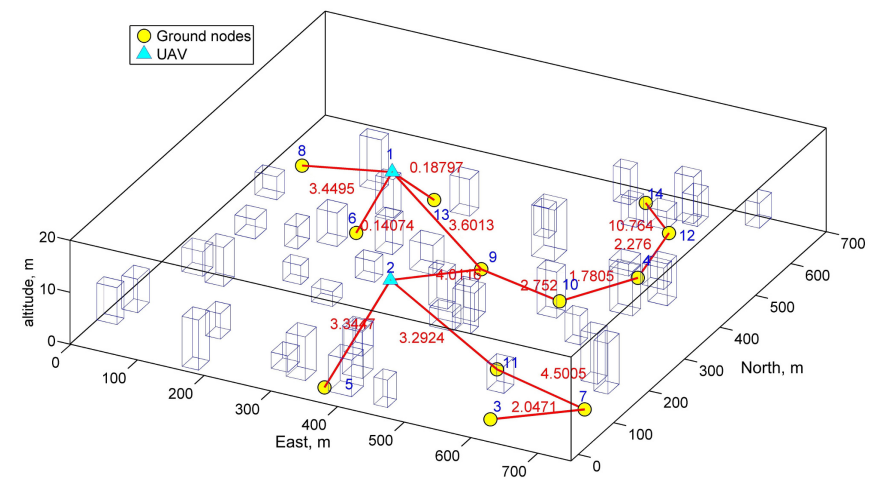

Fig. 1. Sample output of proposed algorithm. The yellow dots represent ground nodes, the blue triangles are UAVs and blue wire-frame shows buildings.

Figure 2 shows a simplified flow chart of the proposed algorithm. The proposed algorithm uses the particle swarm optimisation method with known positions of ground nodes and buildings to determine the best position of relay UAVs for successful communication. The algorithm starts from obtaining the position of ground nodes and relevant building from a planned or online mission and a known urban map. Wireless communication model and requirements are established at the initial phase. Then, communication quality (cost) between nodes including the relay UAV is computed while considering the communication range, signal-to-noise ratio and line-of-sight (LOS) obstruction by buildings. By the optimisation process using a certain communication performance metric, the minimum spanning tree (MST) of the communication network and corresponding optimal relay UAV position is determined. If the desired communication performance of the entire team is not met, more UAVs are added into optimisation and the above process is repeated until obtaining satisfactory performance. The details of each step are explained in the next section.

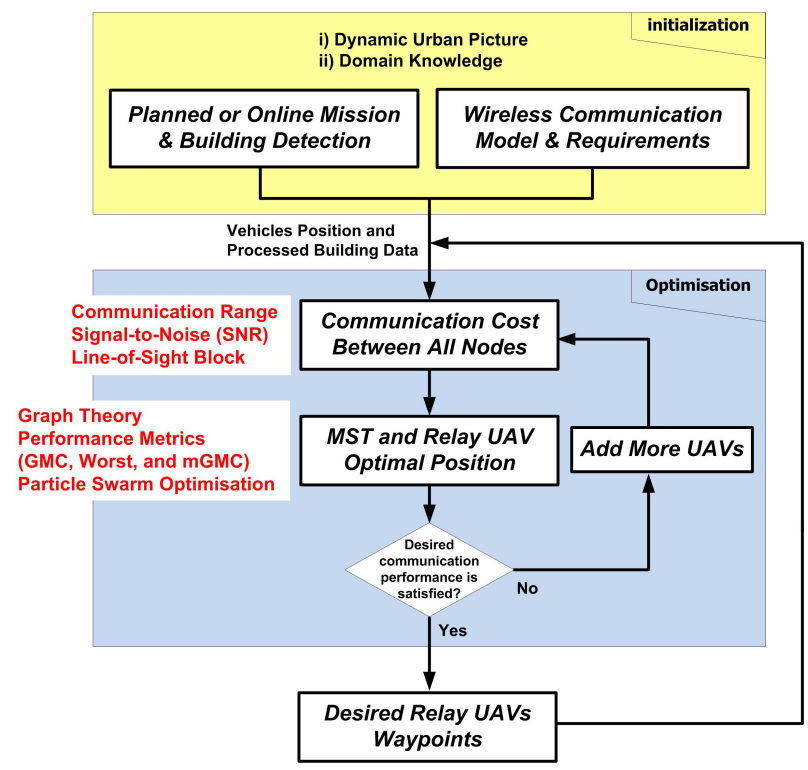

Fig. 2. Flow chart of the proposed communication-aware UAVs position finding algorithm

\section{Optimal Relay UAV POSITIONING}

\section{A. Communication Model}

To find the optimal UAVs positions, communication quality have to be modelled first. In this work, an open space communication model is considered, and extra penalties for the line-of-sight (LOS) block by buildings and UAV flight height are introduced. In particular, a realistic wireless channel propagation communication model based on the work of [7], [8] is utilised. In this model, node $i$ (be it the UAV or the ground vehicle) transmits a signal with the power of $P_{i}$ and observing noise $\sigma_{i}^{2}$ while receiving signals from other nodes. The received signal-to-noise ration (SNR) $\Gamma_{i j}$ for a signal transmitted from the $i$-th node and received by $j$-th node is given as:

$$
\Gamma_{i j}=\frac{P_{i} G_{i j}}{\sigma_{i}^{2}}
$$

where $G_{i j}$ is a channel gain and can be expressed as:

$$
G_{i j}=\frac{C_{i j}\left|h_{i j}\right|^{2}}{D_{i j}^{\alpha}}
$$

where $C_{i j}$ is a constant accounting for antenna gains and shadowing, $h_{i j}$ is responsible for multipath fading, $\alpha$ is a path loss factor and $D_{i j}$ is the distance between the two 
nodes. For a successful message transmission, the SNR needs to be higher than the required minimal link quality $\gamma$. Also, if Rayleigh fading is assumed for a fading model i.e. $h_{i j}$ is the complex Gaussian with zero mean and unit variance, then the probability of successful transmission from $i$-th node to $j$-th node is equal to:

$$
P_{r}^{i j}\left(\Gamma_{i j} \geq \gamma\right)=\exp \left(-\frac{\sigma_{j}^{2} \gamma D_{i j}^{\alpha}}{C_{i j} P_{i}}\right)
$$

Currently effects like diffractions and reflections of a wireless signal due to presence of buildings are not considered to allow for quick and efficient computation.

As more nodes (vehicles) are involved in the scenario, the communication network complexity increases significantly. Thus, sharing of information efficiently becomes a problem. To mitigate this issue, the minimum spanning tree (MST) is used to find the highest probability of successful transmission among nodes in the team using the minimum number of possible links. The MST is defined as subset of graph where all nodes are connected but there is no loops and having a minimum (or at least same as minimum, as there can be several minimum spanning tree in a single scenario) sum of edge weights [13]. In this work, the weighted edge cost between the two nodes (i.e. $i$ and $j$ ) is defined by using the above probability of successful transmission as:

$$
W_{i j}^{o}=-\ln \left(P_{r}^{i j}\right)
$$

Note that, the lower the probability is, the higher the cost is, and this weight will be used as a optimisation cost to be minimised.

In order to account for buildings in an urban environment, an additional weight is considered. This weight is set relatively high to discourage connections through the buildings where the LOS is blocked as they reduce communication quality significantly as:

$$
W_{i j}^{b}= \begin{cases}W_{i j}^{p b} & \text { if the LOS is blocked } \\ 0, & \text { otherwise }\end{cases}
$$

where $W_{i j}^{p b}$ is a penalty cost due to LOS block. For LOS block detection, the Matlab toolbox written in INRA was used [14]. Although this cost is relatively simple to compute, the non-smooth $W_{i j}^{b}$ due to building block can make the solution space highly non-convex containing many local minima, as illustrated in Fig. 3(a). Note that this figure is used only for the illustration purpose showing a communication performance function of the global message connectivity metric when using a single relay UAV at different grid locations at a constant altitude (which will be explained later in detail). To avoid having too many local minima, a new weight is introduced by using the length of LOS block $l_{b}$ inside the building (i.e. the length of the intersection/overlap between the LOS and the obstructing building as illustrated in Fig. 4) as:

$$
W_{i j}^{b}= \begin{cases}W_{i j}^{p b} l_{b} & \text { if the LOS is blocked } \\ 0, & \text { otherwise }\end{cases}
$$

where $l_{b}$ is the distance of LOS intersection with obstructing building and $W_{i j}^{p b}$ is a base cost. As can be clearly seen in Fig. 3(b), the communication performance function is much smoother compared to the previous weight, which helps the optimisation process.

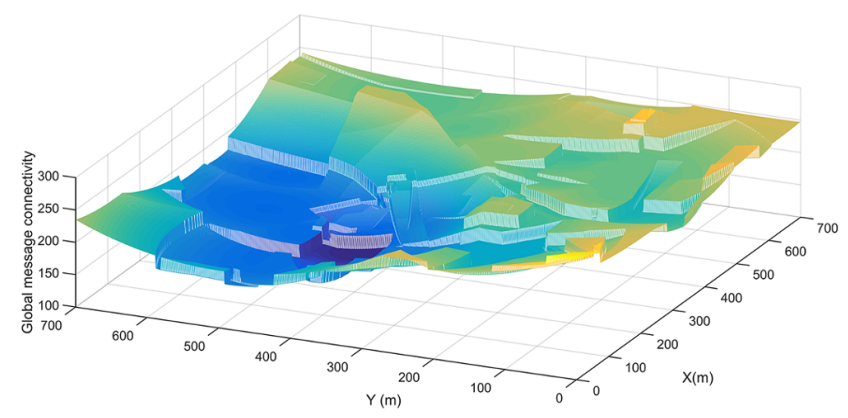

(a) Using a non-smooth weight due to discrete LOS obstruction

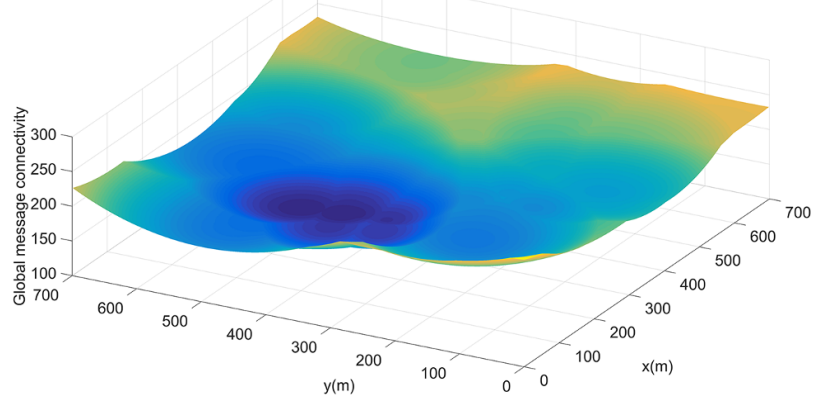

(b) Using a new weight considering the length of overlap

Fig. 3. Surface plot for the communication performance function at different relay UAV locations.

Fig. 4. Illustration of new weight computation. The case on the left would result in a higher cost of the two since the longer portion of the LOS line overlaps with the building.

Another additional weight is due to the UAV flight height restriction. Since the communication probability depends on the distance between nodes as in Eq. (3), it is likely that UAV's optimal position would be dangerously close to the ground and buildings around in order to maximise communication quality. To stop UAV approaching the ground, the minimum allowable UAV height needs to be imposed as a penalty weight as:

$$
W_{i j}^{h}= \begin{cases}W_{i j}^{p h}, & \text { if below minimum height } \\ 0, & \text { otherwise }\end{cases}
$$

where $W_{i j}^{p h}$ is a penalty cost due to the height restriction. It is worthwhile noting that this weight is not related to the communication in a strict sense; it only serves as a barrier to prevent UAVs from flying too low.

Finally, the total communication weight, $W_{i j}^{t}$, can then be expressed as:

$$
W_{i j}^{t}=W_{i j}^{o}+W_{i j}^{b}+W_{i j}^{h} .
$$




\section{B. Communication Performance Metrics}

The optimal UAV position which maximise the communication performance of the team will be different depending on the performance metric (index) used in the optimisation. In this paper, three communication performance metrics are considered: the global message connectivity (GMC), the worst case connectivity (WCC), and the modified global message connectivity (mGMC).

a) Global message connectivity: The GMC is defined as a probability of message being successfully transmitted to all nodes within the minimum spanning tree (MST) [8]. With known positions of all UAVs and ground nodes, the MST can be constructed using previously defined weight $W_{i j}^{t}$ in Eq. (8) for $n$ UAVs and $m$ ground nodes. Let the matrix $A^{\prime} \in R^{(n+m) \times(n+m)}$ represent the adjacency matrix of the MST for a given configuration, then $A_{i j}^{\prime}=1$ if the link from node $i$ to node $j$ is the part of the MST, and $A_{i j}^{\prime}=0$, otherwise. As the sum of the weights in MST is equivalent to the overall probability that a message is successfully transmitted, the performance index is simply equal to the sum of all connections within MST and can be denoted as:

$$
J_{G M C}\left(\overline{\mathbf{x}}^{p o s}, \overline{\mathbf{x}}^{g, p o s}\right)=\sum_{i=1}^{n+m} \sum_{j=1}^{n+m} A_{i j}^{\prime} W_{i j}^{t} .
$$

Note that $A^{\prime}$ and $W^{t}$ are dependent on 3-D location of UAVs and ground nodes, which can be represented as $\overline{\mathbf{x}}^{\text {pos }} \in$ $R^{3 \times n}$ and $\overline{\mathbf{x}}^{g, \text { pos }} \in R^{3 \times m}$, respectively. Thus, the UAV position can be found by minimising the performance index $\left(J_{G M C}\left(\overline{\mathbf{x}}^{p o s}, \overline{\mathbf{x}}^{g, p o s}\right)\right)$ for the GMC with respect to UAV locations $\overline{\mathbf{x}}^{\text {pos }}$ as:

$$
\min _{\overline{\mathbf{x}}^{p o s}} J_{G M C}\left(\overline{\mathbf{x}}^{p o s}, \overline{\mathbf{x}}^{g, p o s}\right)=\min _{\overline{\mathbf{x}}^{p o s}} \sum_{i=1}^{n+m} \sum_{j=1}^{n+m} A_{i j}^{\prime} W_{i j}^{t} .
$$

Figure 5 illustrates how to compute the GMC cost. The cost is a sum of all weighted connections, and this metric is focused on improving overall communication performance of the network, rather than focusing on specific links.

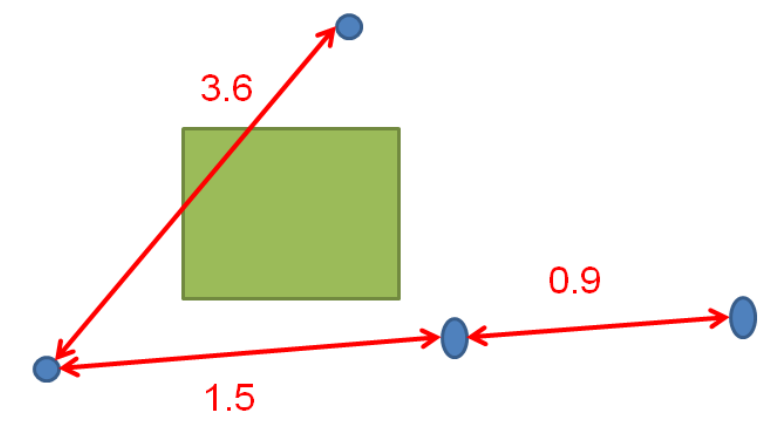

Cost function $=6$

Fig. 5. Cost function with GMC used explained on simple example. The green square is a building, blue circles are nodes and blue lines is an communication MST b) Worst case connectivity: The WCC is represented as the link with the lowest probability of successful communication of all the links within the MST [8]. Thus, the UAV positioning problem can be formulated as minimising the weight of the worst MST link with respect to UAV locations $\overline{\mathbf{x}}^{\text {pos }}$ as:

$$
\min _{\overline{\mathbf{x}}^{p o s}} J_{W C C}\left(\overline{\mathbf{x}}^{\text {pos }}, \overline{\mathbf{x}}^{g, p o s}\right)=\min _{\overline{\mathbf{x}}^{p o s}}\left(\max _{\forall i, j \in \mathbb{M}}\left(W_{i j}^{t} A_{i j}^{\prime}\right)\right)
$$

where $\mathbb{M}=\{1, \cdots, n+m\}$ denotes a set which includes all the nodes in the network (i.e. $m$ UAVs and $n$ ground nodes). For this metric, the cost is the worst connection of the network as illustrated in Fig. 7, and the UAV is positioned so that this connection is improved. This metric is very efficient at ensuring the network connectivity between several disconnected groups of nodes.

c) Modified global message connectivity: Note that the WCC focuses only on improving one worst connection while the GMC improves overall communication performance of the networked team but does not necessarily improve the poor connections (including the worst). If there are requirements for both overall and individual (i.e. between pairs of nodes) communication quality, neither of metrics explicitly satisfy requirements. To this end, the mGMC metric is designed as a compromise between previously discussed the GMC and the WCC. The mGMC metric uses a constant $\beta$ as the number of weak connections in the initial MST (without UAVs) to be improved. This specific number of weak connections can be obtained by defining the desirable minimum communication probability $P_{d}$ of the network.

Firstly, from the desirable probability, the corresponding weight can be calculate as:

$$
W^{d}=-\ln \left(P_{d}\right)
$$

$W^{d}$ is then compared with $W_{i j}^{t}$ for all $i, j \in \mathbb{M}$ in order to find weaker connections than the desired communication quality (probability) in the following manner:

$$
W_{i j}^{t c}= \begin{cases}1, & \text { if } W_{i j}^{t} A_{i j}^{\prime}>W^{d} \\ 0, & \text { otherwise }\end{cases}
$$

where $W^{t c} \in \mathbb{R}^{(n+m) \times(n+m)}$. Lastly, the $\beta$ can be computed as:

$$
\beta=\sum_{i=1}^{n+m} \sum_{j=1}^{n+m} W_{i j}^{t c}
$$

The computed $\beta$ is used in the mGMC metric, by firstly rearranging the connectivity matrix into a vector:

$$
W^{t s}=\operatorname{vec}\left(W^{t} A^{\prime}\right)
$$

where $W^{t s} \in \mathbb{R}^{(n+m)^{2} \times 1}$. This vector is sorted in a descending order and stored as $W^{t s}$. The mGMC cost can then be defined as:

$$
\min _{\overline{\mathbf{x}}^{p o s}} J_{m G M C}\left(\overline{\mathbf{x}}^{\text {pos }}, \overline{\mathbf{x}}^{g, p o s}\right)=\min _{\overline{\mathbf{x}}^{\text {pos }}} \sum_{k=1}^{\beta} W_{k}^{t s}
$$

The mGMC focuses neither on overall communication performance nor the worst connection, but rather on improving 


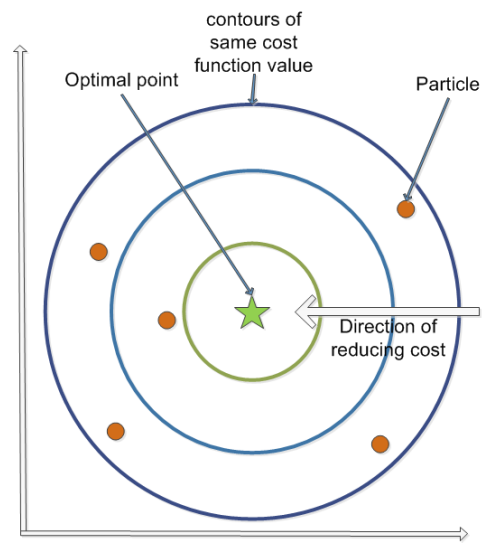

(a) Initial random positions

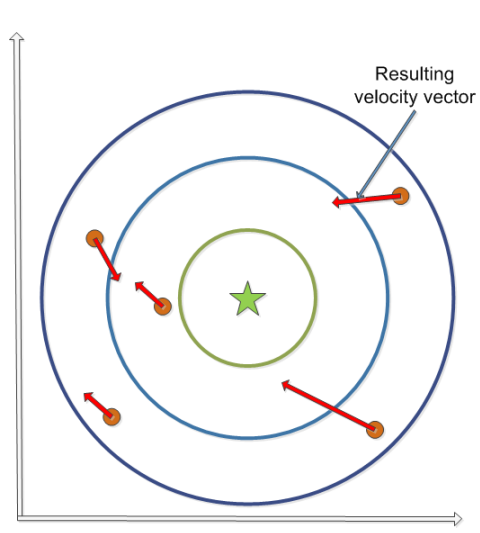

(b) Moving towards the velocity vector

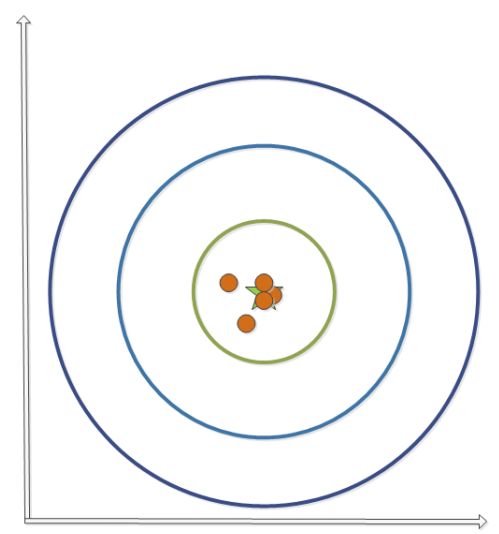

(c) Final particles positions

Fig. 6. Illustration of the particle swarm optimisation process.

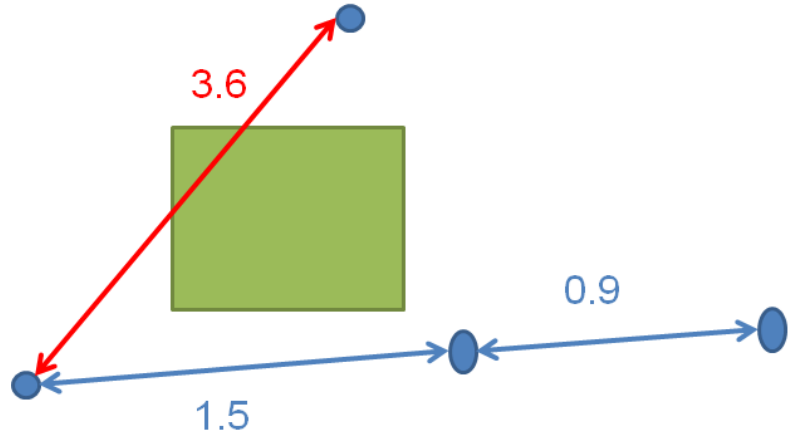

Cost function $=3.6$

Fig. 7. Cost function with WCC used explained on simple example

a certain number of connections depending on user requirements. The computation of the metric is illustrated in a simple example in Fig. 8. For this case, as $\beta$ is 2 as $W^{d}=1$, the total mGMC metric cost is equal to 5.1 .

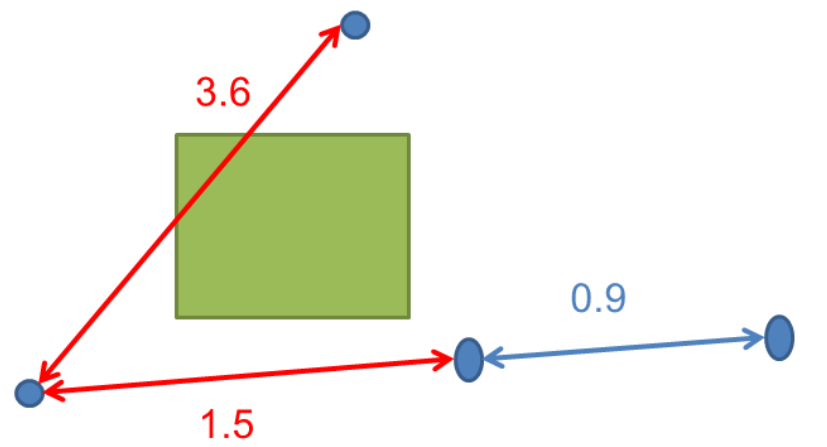

For $W^{d}=1, \beta=2$

Cost function $=5.1$

Fig. 8. Cost function with the mGMC used explained on simple example

\section{Optimisation}

A particle swarm optimisation (PSO) technique [15] is used to find the optimal position of relay UAVs which minimise the communication performance metrics defined as above. The PSO is chosen as it has the ability to solve complex non-smooth and non-convex problems. In the PSO algorithm, initially, particles are randomly spread over the problem space and they evaluate their cost functions. The next position is determined by computing velocity vector in each dimension of the problem as:

$$
\begin{aligned}
v_{i, d}= & r_{1} \omega v_{i-1, d}+\varepsilon r_{2}\left(X_{i b, i-1, d}-X_{c, i-1, d}\right) \\
& +\varepsilon r_{3}\left(X_{g b, i-1, d}-X_{c, i-1, d}\right)
\end{aligned}
$$

where $d$ denotes a problem space dimension, $i$ represents the iteration step, $\omega$ represents the inertia of the particle, and $\varepsilon$ is a correction factor. $X_{i b}, X_{g b}$ and $X_{c}$ represent the best position of a given particle, the global best position (considering all particles) and the current position of the particle, respectively. $v_{i, d}$ is the particle velocity, and $r_{1}, r_{2}, r_{3}$ are random numbers from zero to one. The optimisation process is illustrated in Fig. 6. The calculation stops once the convergence condition (e.g. the maximum number of iterations) is satisfied.

\section{Numerical Simulation AND Indoor Flight EXPERIMENT RESULTS}

Numerical simulations are performed to validate the proposed approach and compare three communication performance metrics one another. Communication parameters are taken from commercial-off-the-shelf Ubiquity pico station M2. Table I shows parameters of this kind of a low-cost and low-power communication equipment.

TABLE I

RADIO COMMUNICATION PARAMETER

\begin{tabular}{ccc}
\hline Parameter & Value & Unit \\
\hline \hline Transmission power $\left(P_{i}\right)$ & 0.01 & $\mathrm{~W}$ \\
Noise power $\left(\sigma^{2}\right)$ & $1 \times 10^{-9}$ & $\mathrm{~W}$ \\
Max communication range & 300 & $\mathrm{~m}$ \\
Attenuation factor $(\alpha)$ & 3 & $\mathrm{n} / \mathrm{a}$ \\
Antenna gain $C$ for $(\mathrm{UGV}, \mathrm{UAV})$ & $(1,1)$ & $\mathrm{n} / \mathrm{a}$ \\
\hline
\end{tabular}




\section{A. A Sample Scenario}

For a sample scenario, two relay UAVs and twelve ground nodes are used with 30 buildings in the urban environment. Figure 9 shows the optimal deployment results for relay UAVs using different communication performance metrics in the optimisation process. For $J_{m G M C}$ computation, $\beta$ is set to three (or equivalently $W^{d}=20$ ) as a desired communication requirement.

Firstly, Fig. 9(a) shows the minimum spanning tree (MST) connection (shown as red lines) without using any UAVs. Many of connections are weak due to line-of-sight (LOS) block by buildings and relatively large distances between nodes. In particular, the weakest connection is between nodes 11 and 12 (represented by the green line) with the weighted edge cost of $J_{W C C}=39.01$. The sum of all connection (termed as the global cost, hereafter) in the MST for this sample scenario is $J_{G M C}=281.4$, while the sum of $\beta$ worst connections is $J_{m G M C}=167.2$.

Figure 9(b) shows the result of using the global message connectivity (GMC) as the performance metric in the optimisation. As expected, the global cost was improved significantly as $J_{G M C}=154.12$. However, it is worth noting that, even though the connection between nodes 6 and 12 is very weak ( $W_{612}^{t}=20.13$, which is above desired communication quality), it is not improved by using the GMC. This is because placing the UAV in between nodes 6 and 12 would improve only one connection while the purpose of the GMC metric is to improve communication quality of all connections globally.

Using the worst case connectivity (WCC) as the performance metric is shown in Fig. 9(c). There are two notable connections improved by this metric: between nodes 11 and 12 and nodes 6 and 12. As a result, the worst connection within the MST is now 16.3 instead of 20.13 as in the previous case. However, the global cost is the worst among three metrics as $J_{G M C}=186.04$. This is because this metric does not consider global communication performance while focusing only on improving the one worst connection.

Finally, the proposed metric, the modified global message connectivity (mGMC) is used on the same scenario with $\beta=3$ as shown in Fig. 9(d). For this performance metric, the global cost is $J_{G M C}=163.1$ which lies in the middle between the GMC (154.12) and the WCC (186.04) metrics, and the worst connection cost $J_{W C C}=16.3$ is the same as using the WCC as a metric. For the sum of three worst connections, this metric provides the best result $J_{m G M C}=82.4$ among three different metrics. Thus, the proposed mGMC metric satisfies the desired communication requirement (i.e. $\left.W^{d}=20\right)$ as well as provides the reasonable global communication performance.

\section{B. Monte Carlo Simulation}

Monte Carlo simulations are performed to confirm the aforementioned trends. The results presented above are averaged over 30 independent runs. Figure 10(a) shows how the global cost (i.e. $J_{G M C}$ ) changed by using three performance metrics with the increasing number of UAVs. Generally, the

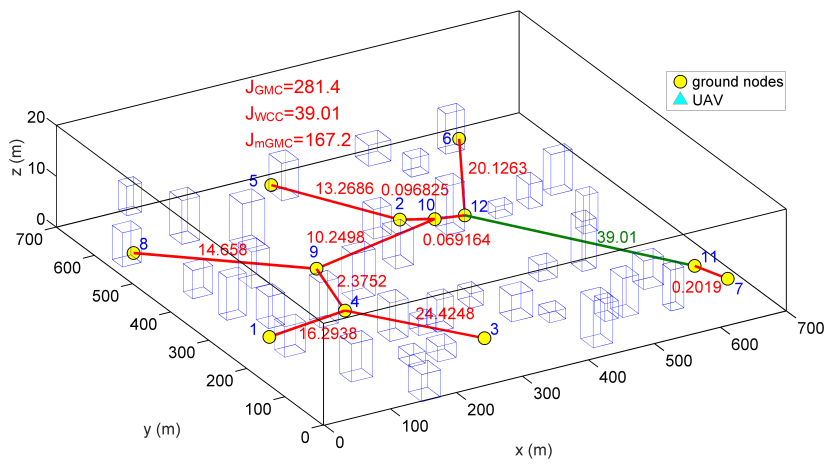

(a) MST without UAVs

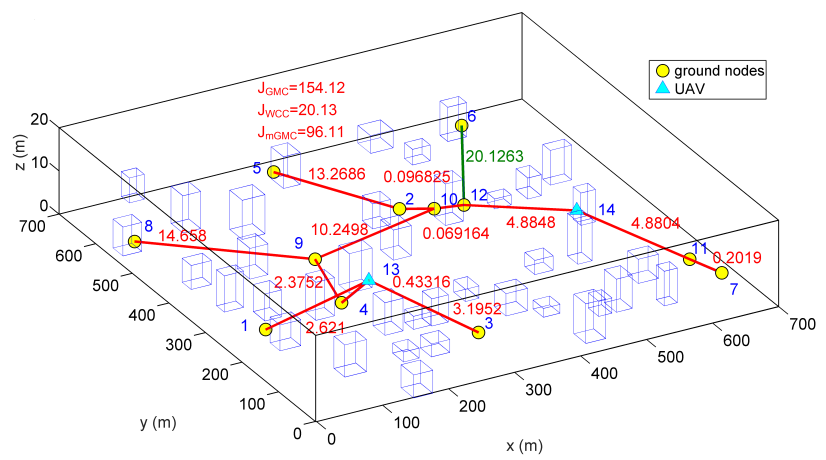

(b) Using the GMC with two UAVs

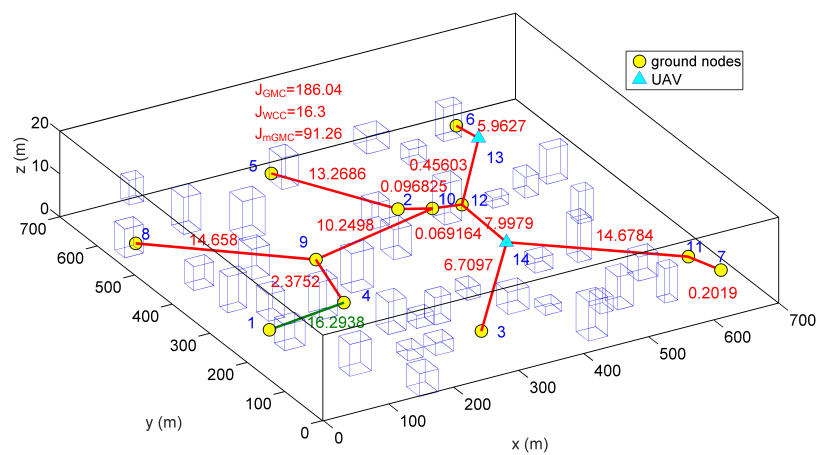

(c) Using the WCC with two UAVs

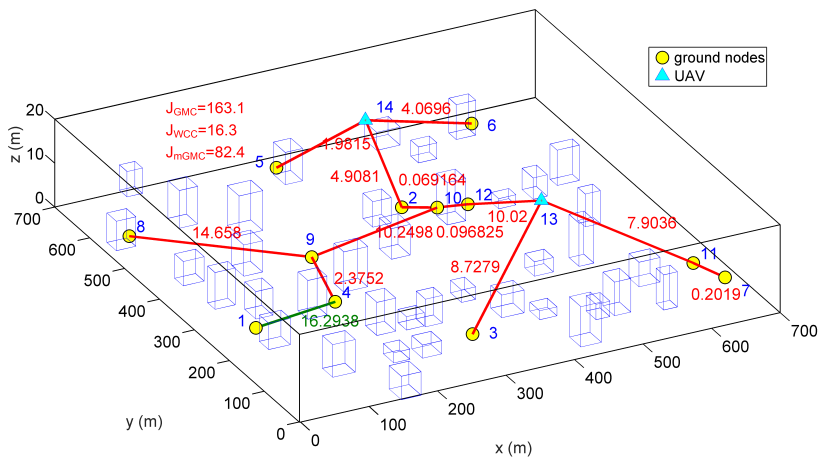

(d) Using the mGMC with two UAVs

Fig. 9. Optimal deployment results for relay UAVs using different communication performance metrics. 


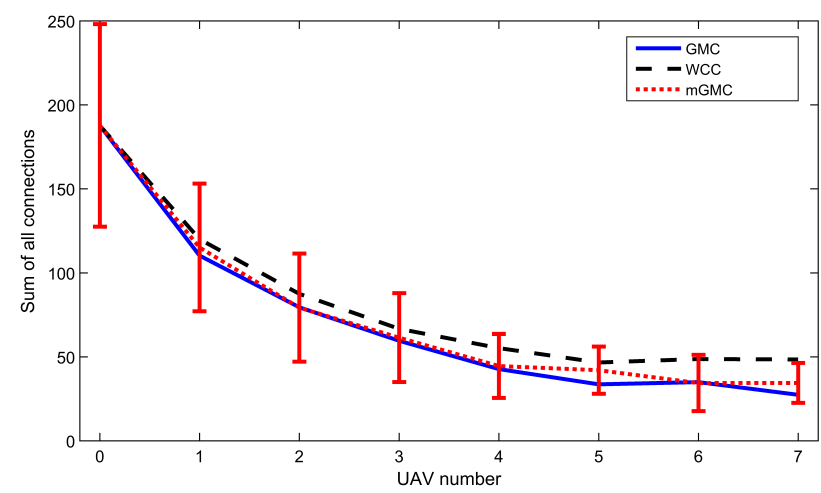

(a) $J_{G M C}$ cost

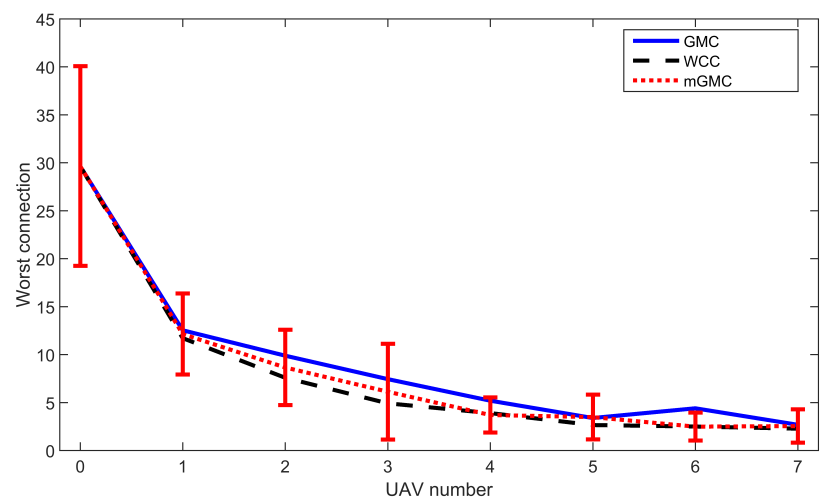

(b) $J_{W C C}$ cost

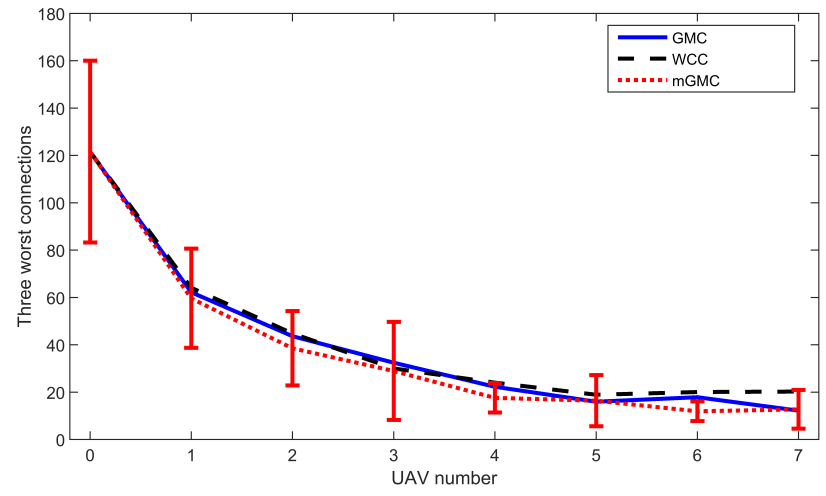

(c) $J_{m G M C}$ cost

Fig. 10. Monte Carlo simulation results using three different communication performance metrics with different number of UAVs.

GMC metric (represented as a blue solid line) shows the best result, but the mGMC metric result is not much worse, especially for the small number of UAVs. This is because with the small number of UAVs there are a significant number of weak connections, and improving those can have a dramatic impact on the global cost. The WCC shows the worst performance in terms of the global cost similar to a sample scenario case. Figure 10(b), with the worst connection as a metric, shows the opposite situation to the GMC as in Fig. 10(a). The WCC has the best performance in terms of the worst connection (i.e. $J_{W C C}$ ), and shows the faster reduction of worst connection cost of all three metrics while the GMC metric shows the worst performance.
From Fig. 10(c), it can be observed that, for $\beta=3$ worst connections, the mGMC shows the best performance in terms of the sum of three $(\beta)$ worst connections, while the other two metrics provides worse performance.

\section{Indoor Flight Experiment}

To validate the algorithm, an indoor flight experiment ${ }^{1}$ was performed using a quad rotor UAV and three unmanned ground vehicles (UGVs) in an artificial urban environment. Each vehicle is equipped with a Raspberry pi B+ integrated with a commercial off-the-shelf autopilot (ardupilot 2.6 [16]) for on-board position control. The proposed optimal position finding algorithm is run on the external computer (i.e. ground control system), and computed desired positions are send to the UAV via Wi-Fi network. Communication amongst UGVs and UAV in experiment is purely virtual due to insignificant signal strength loss from artificial buildings. A Vicon motion tracking [17] system is used to provide accurate position information of the vehicle in an indoor environment. For the experiment, the mGMC metric is used as a performance metric with $\beta=3$, and there are three set of waypoints for the UGVs to visit in the mission scenario.

Figure 11(a) shows the optimal position of the UAV for the initial waypoints of UGVs with red lines representing the MST. In this case, the UAV is required to function as a relay between all three UGVs as the line-of-sight (LOS) between all of them is obstructed by buildings. For the next UGV waypoints shown in Fig. 11(b), the UGV in the middle lane and the UGV in the right lane changed their position, which makes the LOS to each other blocked. The UAV ensures communication between those two vehicles. As the other UGV (in the left lane) has a direct LOS to the middle lane UGV, those two are directly connected, not through the relay UAV. For the final UGV waypoints shown in Fig. 11(c), the UAV moves to the position which improves communication between UGVs in the middle lane and the right lane as the LOS is blocked.

\section{CONClusions And Future Work}

This paper has discussed optimal placement of relay UAVs to improve communication performance of the ground mobile network in an urban environment. The communication performance metric, termed as the mGMC, was proposed to find the optimal UAV position which satisfies the desired communication requirement of the network. Numerical simulations showed significant improvements of the probability of successful communication using relay UAVs, and an indoor flight test showed potential for the near real-time algorithm execution. One of the main improvements will be the implementation of a more accurate urban communication networking modelling, possibly combining modeland measurement-based approaches mentioned in Section I. Another future extension will be a decentralised scheme where UAVs do not have full knowledge of every vehicle positions.

${ }^{1}$ A short video including the indoor flight test and some numerical simulation results is provided at the following link: https://dl.dropboxusercontent.com/u/42733842/UAV_Relay_Movie.mp4 


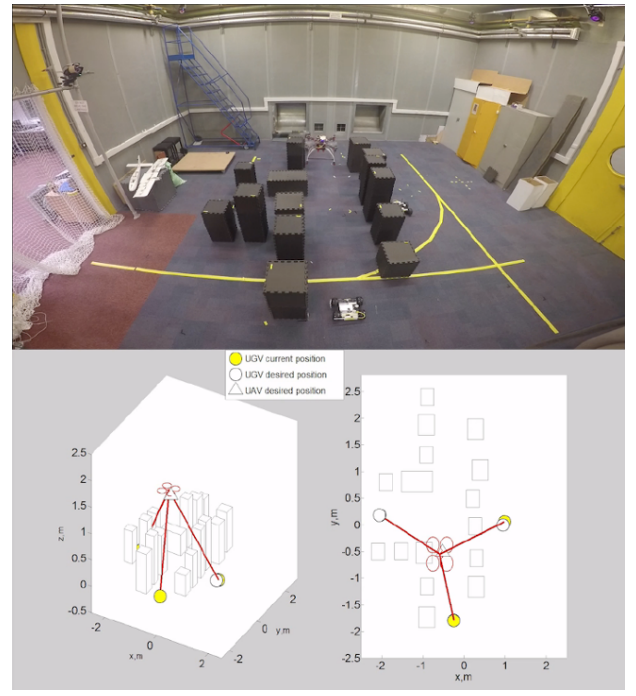

(a) First UGV waypoints

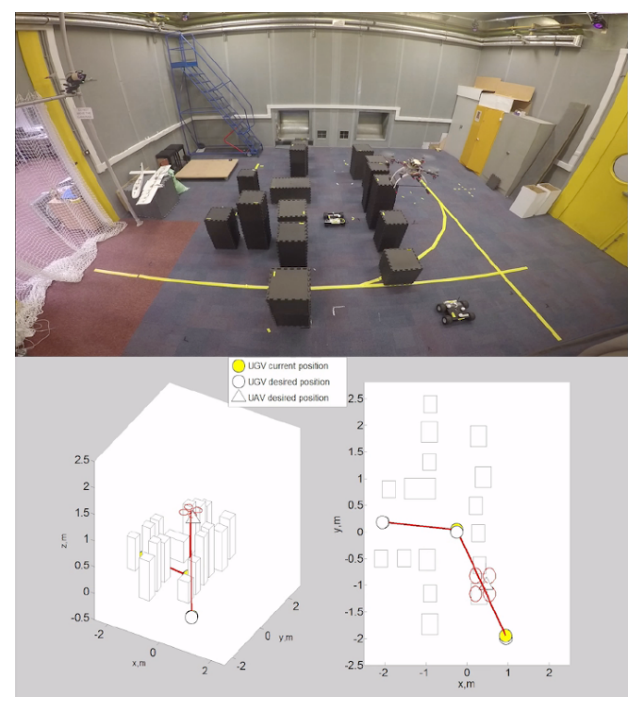

(b) Second UGV waypoints

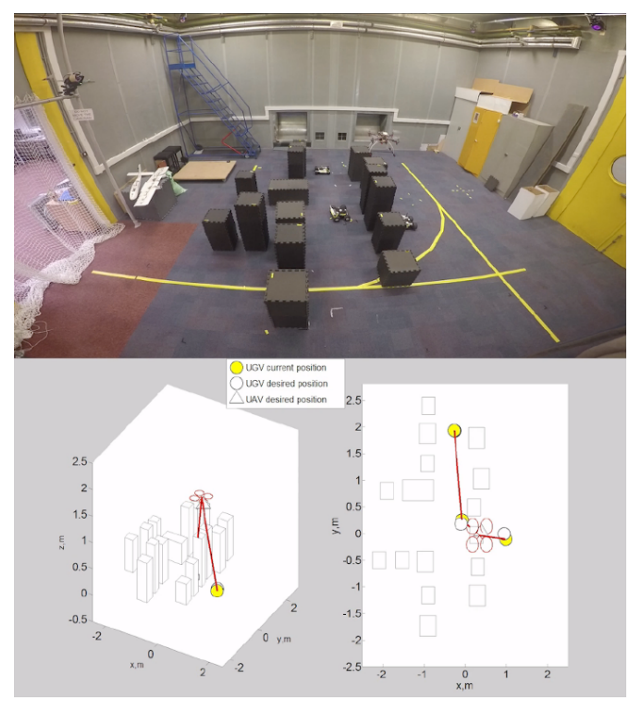

(c) Third UGV waypoints

\section{REFERENCES}

[1] D. G. Press, "Urban warfare: Options, problems and the future," Janaury 1999. [Online]. Available: http://web.mit.edu/ssp/publications/ conf_series/urbanwarfare/urbanwarfare.html

[2] H.-S. Shin and P. Segui-Gasco, "UAV Swarms: Decision-Making Paradigms," Encyclopedia of Aerospace Engineering, pp. 1-13, 2014. [Online]. Available: http://doi.wiley.com/10.1002/9780470686652. eae 273

[3] H. Oh, S. Kim, H. Shin, P. Ladosz, and A. Tsourdos, "Communicationaware convoy following guidance for UAVs in a complex urban environment," in The 24th Mediterranean Conference on Control and Automation, Athens, Greece, 2016.

[4] P. Basu, J. Redi, and V. Shurbanov, "Coordinated flocking of UAVs for improved connectivity of mobile ground nodes," IEEE MILCOM 2004. Military Communications Conference, 2004., vol. 3, pp. 16281634,2004

[5] A. S. Ibrahim, K. G. Seddik, and K. J. Ray Liu, "Improving connectivity via relays deployment in wireless sensor networks," GLOBECOM - IEEE Global Telecommunications Conference, pp. 1159-1163, 2007.

[6] D.-T. Ho, E. I. Grotli, P. B. Sujit, T. A. Johansen, and J. B. De Sousa, "Performance evaluation of cooperative relay and Particle Swarm Optimization path planning for UAV and wireless sensor network," 2013 IEEE Globecom Workshops (GC Wkshps), pp. 1403-1408, 2013. [Online]. Available: http://ieeexplore.ieee.org/lpdocs/epic03/wrapper. htm?arnumber $=6825191$

[7] S. Kim, H. Oh, J. Suk, and A. Tsourdos, "Coordinated trajectory planning for efficient communication relay using multiple UAVs,' Control Engineering Practice, vol. 29, pp. 42-49, 2014. [Online]. Available: http://dx.doi.org/10.1016/j.conengprac.2014.04.003

[8] Z. Han, a. L. Swindlehurst, and K. J. R. Liu, "Optimization of MANET Connectivity Via Smart Deployment / Movement of Unmanned Air Vehicles," Ieee Transactions on Vehicular Technology, Vol. 58, No. 7, September 2009, vol. 58, no. 7, pp. 3533-3546, 2009.

[9] E. P. D. Freitas, T. Heimfarth, I. F. Netto, C. E. Lino, C. E. Pereira, A. M. Ferreira, F. R. Wagner, and T. Larsson, "UAV relay network to support WSN connectivity," 2010 International Congress on Ultra Modern Telecommunications and Control Systems and Workshops, ICUMT 2010, pp. 309-314, 2010.

[10] D. J. Lee and K. Andersson, "Hybrid control of long-endurance aerial robotic vehicles for wireless sensor networks," International Journal of Advanced Robotic Systems, vol. 8, no. 2, pp. 101-113, 2011.

[11] C. Dixon and E. W. Frew, "Optimizing cascaded chains of unmanned aircraft acting as communication relays," IEEE Journal on Selected Areas in Communications, vol. 30, no. 5, pp. 883-898, 2012.

[12] D. H. Choi, B. H. Jung, and D. K. Sung, "Low-complexity Maneuvering Control of a UAV-based Relay without Location Information of Mobile Ground Nodes," Computers and Communication (ISCC), 2014 IEEE Symposium on, pp. 1 - 6, 2014.

[13] R. Sedgewick and K. Wayne, "Minimum spanning trees," 2015. [Online]. Available: http://algs4.cs.princeton.edu/43mst/

[14] D. Legland, "geom3d," June 2009. [Online]. Available: http: //www.mathworks.com/matlabcentral/fileexchange/24484-geom3d

[15] J. Kennedy and R. Eberhart, "Particle swarm optimization," Neural Networks, 1995. Proceedings., IEEE International Conference on, vol. 4, pp. 1942-1948 vol.4, 1995.

[16] 3DRrobotics, http://ardupilot.com/

[17] Vicon Motion systems, http://vicon.com/.

Fig. 11. Snapshots from the experiment which finds the optimal UAV positions and the MST (red lines) for UGV waypoints. 